\title{
Uncoupling of liquid and solid retention times in anaerobic digestion of catering wastes
}

\author{
M.A. Climenhaga* and C.J. Banks* \\ *School of Civil Engineering and the Environment, University of Southampton, Southampton SO17 1BJ, UK \\ (E-mail: mac2@soton.ac.uk; cjb@soton.ac.uk)
}

\begin{abstract}
Source-separated food wastes collected from a university campus catering facility were processed in bench-scale anaerobic digesters. The feedstock contained a varied mix of fruits, vegetables, meats and fried foods. Two modes of digestion were compared. The first was hydraulic flush (HF) mode, in which liquids were flushed through the reactor on a retention time of 25 days while solids were maintained on an extended retention time of over 150 days. The converse was a solids wastage (SW) mode, in which liquid retention time was over 150 days, and solids were wasted to maintain a retention time of 25 days. SW reactors exhibited methanogenic failure after approximately 45 days. HF reactors, in contrast, maintained stable digestion for a period of 100 days, and were robust enough to recover from a thermal shock applied over a three-day period in which the temperature was increased from $35^{\circ} \mathrm{C}$ to $50^{\circ} \mathrm{C}$ between days $105-108$ of the experiment. Stable operation was regained by day 139 and continued until the end of the run on day 150 .
\end{abstract}

Keywords

Anaerobic digestion; solids retention time; hydraulic flush; solid-liquid separation; catering wastes; food wastes

\section{INTRODUCTION}

Anaerobic digestion of organic wastes from domestic and institutional sources is a growing field in Europe (De Baere, 2006) and throughout the world. Socio-political and economic drivers favouring waste management technologies with a positive energy balance and potential to reduce emissions of greenhouse gases are creating favourable conditions for the expansion of anaerobic digestion (Hogg et al., 2007). New market opportunities for anaerobic digestion technologies include the use of the process for feedstocks which are new or for which few commercial examples exist, such as sourceseparated food wastes from food serving establishments. Successful continued development of the technology requires understanding of the particular challenges posed by the different feedstocks that could potentially be processed, and determination of optimal modes of digestion.

One important factor for consideration is the retention time for solids and liquids in the system. In treatment of liquid wastewaters, bioreactors are commonly designed to retain anaerobic biomass solids (e.g. upflow anaerobic sludge blanket, anaerobic filter, expanded granular sludge bed reactor types), as these work with a very short HRT which is less than the maximum growth rate for methanogens, and therefore mechanisms are required to maintain biomass in the reactor. Many solid waste digestion processes, on the other hand, do not uncouple solid and liquid retention times. The hydrolysis of particulate substrate is often rate-limiting (Sanders, 2001) and sets the retention time for both the solid and liquid fractions of these systems (Veeken et al., 2000).

In previous work (Climenhaga and Banks, 2007) on digestion of source-separated food wastes using a CSTR design, the roles of hydraulic retention time and trace element supplementation were investigated. In both systems, trace element supplementation was essential for sustained stable operation. It was also found that extending the hydraulic retention time resulted in higher levels of alkalinity in the system and facilitated more stable operation: bioreactors on a hydraulic retention 
time of 50 days showed stable operation for a longer period than those on a hydraulic retention time of 25 days. The work described here focused on determining whether it was the retention of solids or the retention of liquids that was responsible for this observation, i.e., the relative importance of solids vs. liquids in maintenance of alkalinity and stability of the system.

\section{MATERIALS AND METHODS}

Substrate for the digestion trials was source-separated foodwaste from the main catering facility serving staff and postgraduates at the University of Southampton (Highfield Campus, Southampton UK). Wastes were collected over a period of five days, from all areas of the facility including salad bar (primarily fruit and vegetables), hot food counter (cooked foods including meats and fried foods) and preparation kitchen (peelings, bones and fat trimmings). The wastes were ground in a commercial garbage grinder (S52/010 Waste Disposer, Imperial Machine Company Ltd., Hertfordshire UK) and mixed to form a large composite. The composite was then frozen in $1500 \mathrm{~g}$ portions, stored at $-16{ }^{\circ} \mathrm{C}$ and thawed as needed for use as substrate. Foodwaste was characterised for total solids (TS), volatile solids (VS) and total Kjeldahl nitrogen (TKN) by standard methods (APHA, 2005). Total lipid content was analysed using a Soxhlet extraction (APHA, 2005) using heptane as solvent. Chemical oxygen demand (COD) was measured following a modification of the standard method (APHA, 2005) using larger quantities of the same reagents to analyse a larger sample.

Four bioreactors were constructed from $1000 \mathrm{ml}$ polypropylene centrifuge bottles with a $40 \mathrm{rpm}$ stirrer mounted on the sealable screw cap. The entire stirrer mechanism, including paddle stirrer, could be removed and replaced by a standard screw cap which allowed the reactors to be centrifuged at $4000 \mathrm{rpm}$ (Wifug 4000E centrifuge, Wifug Ltd., Bradford UK) for solid-liquid separation. A diagrammatic representation of the reactor design is shown in Figure 1.

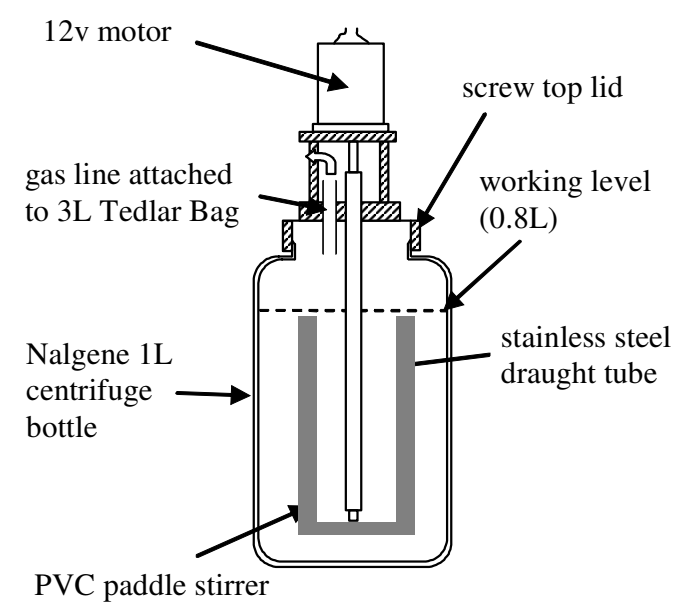

Figure 1: Centrifuge bottle bioreactor.

The reactors were seeded with sludge from a wastewater treatment plant anaerobic digester (Millbrook Sewage Works, Southampton) and operated with uncoupled solid and liquid retention times. Two reactors were operated by conserving all solids in the reactor and periodically flushing a portion of the liquid (Hydraulic Flush, HF), while the other two were operated by retaining all liquid in the reactor but periodically wasting a portion of the solids (Solids Wastage, SW). Solidliquid separation was carried out on a weekly basis, by centrifuging the reactors and decanting the supernatant through 1-mm mesh. A mass equal to $28 \%$ (based on a removal of $4 \%$ per day, 
multiplied by 7 days per week) of either the liquid or solid was removed each week, before recombining the liquid with the solids in the reactor and adding deionised water to bring the reactor back up to its working volume. HF reactors were operated with a hydraulic retention time (HRT) of 25 days, and a solids retention time (SRT) greater than 150 days. SW reactors were operated with HRT greater than 150 days, and SRT 25 days.

Biogas production was measured daily, via water displacement in a volume-calibrated cylindrical gas collector after collection in Tedlar bags (SKC Ltd., Dorset UK). Gas composition samples were withdrawn from the headspace of the reactors once per week, analysed by gas chromatography and compared to a standard mix of $35 \% \mathrm{CO}_{2} / 65 \% \mathrm{CH}_{4}$, on a Varian $\mathrm{CP}-3800$ gas chromatograph with thermal conductivity detector, column temperature $50{ }^{\circ} \mathrm{C}$. Volatile fatty acids (VFA) were measured weekly using a Shimadzu GC-2010 gas chromatograph (Shimadzu Europe Ltd., Manchester UK), with flame ionisation detector and FFAP capillary column SGE model BP-21 (SGE Europe Ltd., Milton Keynes UK), with helium as the carrier gas. Measurement of total ammonia nitrogen (TAN) and total solids were in accordance with standard methods (APHA, 2005). Alkalinity was measured using a three-point titration. While titration to 4.0 provides a measure of the total alkalinity in the system, this endpoint includes both carbonate alkalinity and alkalinity due to the salts of VFA, which have proton-accepting capacity at a low $\mathrm{pH}$ (below 5.75). Therefore, the total alkalinity can be misleading, as it includes alkalinity that does not actually buffer the system in the range needed by methanogens (Lahav et al., 2002). To separate the different buffering, Partial Alkalinity (PA) was measured to $\mathrm{pH} 5.75$, while an endpoint of 4.3 was used to determine Intermediate Alkalinity (IA) due primarily to the salts of VFA (Ripley et al., 1986).

The reactors were run as duplicates, except that one reactor from each pair was supplemented with a trace element mixture on a weekly basis while the other reactor was not. The trace element solution followed the recipe of Gonzalez-Gil et al. (2001). Reactors were kept in a water bath with a constant temperature of $35^{\circ} \mathrm{C}$, except for a thermal shock episode between days 105-108, when temperature in the water bath rose to $50{ }^{\circ} \mathrm{C}$. The organic loading rate (OLR) of $1.45 \mathrm{gVS} \mathrm{l}^{-1} \mathrm{~d}^{-1}$ was constant throughout the trial except for adjustments during a three-week period following the thermal shock.

\section{RESULTS AND DISCUSSION}

Table 1 shows the characteristics of the food waste substrate used. Values are average and standard deviation of six replicates. In addition to being high in volatile solids, the substrate is high in lipid content due to the high level of fried foods in the collected feedstock.

Table 1 Characteristics of food waste substrate

\begin{tabular}{ll}
\hline Parameter & Average \pm Std Deviation \\
\hline Total Solids (\%) & $28.1 \pm 0.25$ \\
Volatile Solids (\% of TS) & $95.5 \pm 0.1$ \\
Total Kjeldahl Nitrogen (\% of TS) & $3.8 \pm 0.2$ \\
Total Lipid Content (\% of TS) & $22.2 \pm 0.2 \%$ \\
Chemical Oxygen Demand ( $\left./ \mathrm{kg}_{\text {fresh weight }}\right)$ & $422 \pm 16$ \\
\hline
\end{tabular}

Digestion results are shown in Figure 2. Though the two sets of reactors were similar in terms of alkalinity, TAN, VFA and specific methane production at the commencement of the study, they had 
diverged significantly after 40 days. Biomass growth in the SW reactors was not sufficient to keep up with removal rate on a 25-day SRT, as shown by the decrease in TS through the trial. These reactors showed an increase in TAN and VFA, as expected since these soluble compounds would be
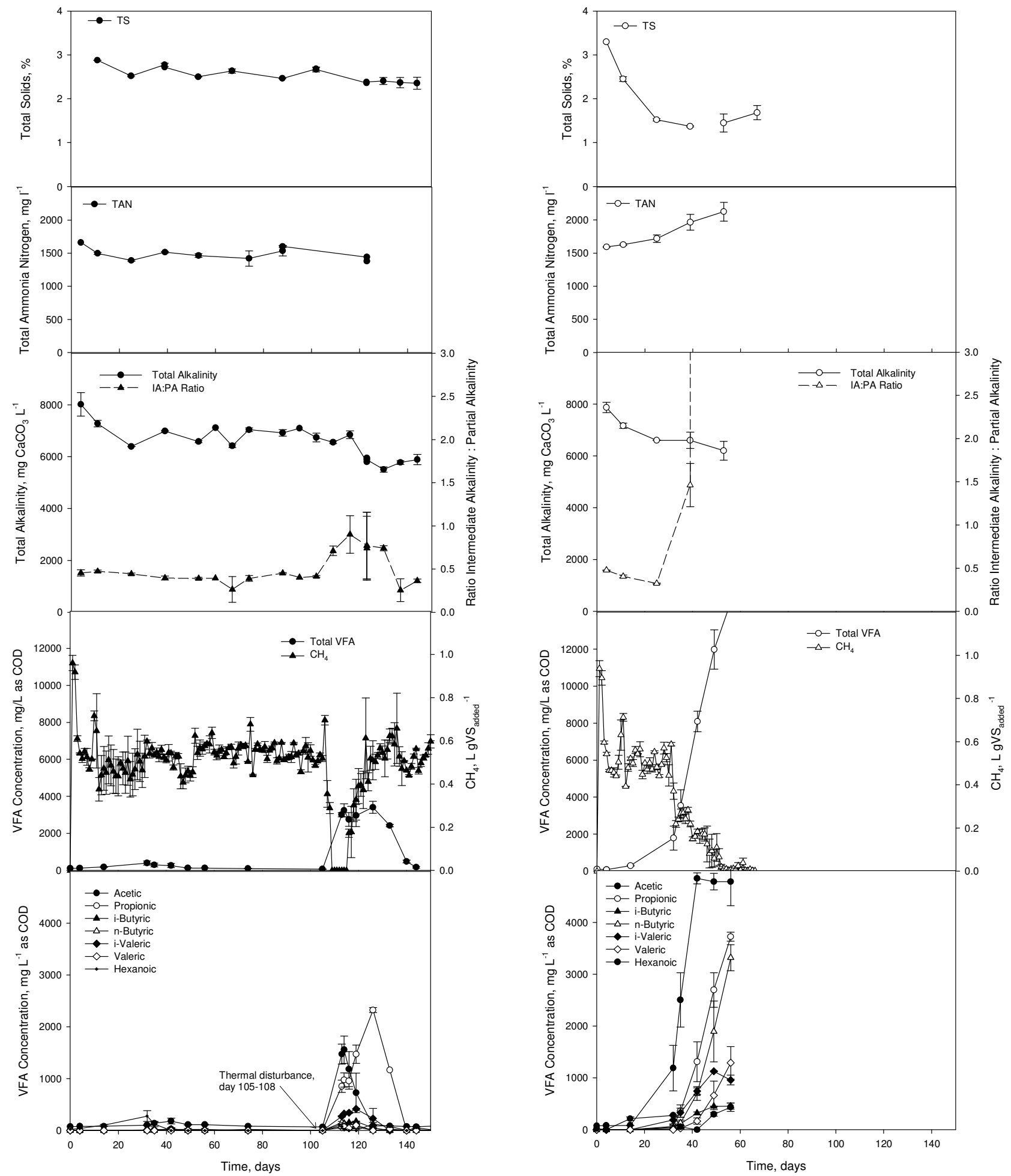

Figure 2: Total solids, total ammonia nitrogen (TAN) concentrations, total alkalinity and ratio of intermediate : partial alkalinity, total VFA, specific methane production, and concentrations of individual VFA for reactors operated in hydraulic flush mode (left) or solids wastage mode (right). Points are average of two reactors, error bars represent range of values between the two reactors. 
retained in the liquid fraction. Although total alkalinity (as titrated to $\mathrm{pH} 4.0$ ) stayed fairly constant, the ratio of intermediate alkalinity to partial alkalinity rose rapidly between days 25-40, indicating the presence of high concentrations of VFA relative to bicarbonates in the system. This is confirmed by the rapid increase in VFA concentrations, ending in methanogenic failure as shown by the cessation of methane production. This was true for both reactors of this pair, regardless of trace element supplementation. Feeding to the SW reactors was stopped at day 67, following a number of days in which no gas was produced and total VFA levels continued to increase.

In contrast, the HF reactors maintained stable levels of TS, TAN, alkalinity and VFA throughout the portion of the trial during which uniform conditions were maintained, a period of 100 days. After this point, the temperature was raised from $35{ }^{\circ} \mathrm{C}$ to $50{ }^{\circ} \mathrm{C}$ from days $105-108$, which resulted in an increase in VFA, indicating an imbalance during which the rate of acidogenesis and acetogenesis exceeded that of methanogenesis. Feeding was stopped for a period of 7 days (days 109-115) following the thermal shock, then resumed initially at full feed for one week (days 116-122) and then at $86 \%$ of full feed for two weeks (days 123-139), by which time VFA levels and methane production had returned to the levels preceding the thermal shock, indicating recovery of the system. The full OLR of $1.45 \mathrm{gVS} \mathrm{l}^{-1} \mathrm{~d}^{-1}$ was then applied for the remainder of the run (days 140150). Figure 2 shows the succession of VFA during the disturbance, in which acetic acid was the first VFA to rise, followed by propionic which continued to rise after acetic acid had returned to its previous levels, before also being consumed. Both HF reactors behaved similarly regardless of the presence or absence of trace element supplementation.

Previously it was found that trace element supplementation was required for stable digestion of this feedstock in CSTR digesters (Climenhaga and Banks, 2007). In the current work, however, regular supplementation with trace elements made no difference to reactor performance - it was not required for hydraulic flush reactors, and was not sufficient to prevent failure for solids wastage reactors. One possible explanation for this is the low solubility of micronutrients such as cobalt, iron and nickel within the $\mathrm{pH}$ range for anaerobic digestion. These metals are likely to adsorb to unhydrolysed particles and microbial cells rather than dissolving, and therefore most of the initial mass of any micronutrient in the reactor would be conserved in the solid fraction. Therefore in the case of the hydraulic flush reactors, micronutrients would stay in the reactor rather than washing out over time with the liquid fraction, while in the case of the solids wasting reactors, micronutrients would be removed with the solids.

In comparing the relative performance of the two systems with extended SRT or extended HRT, some conclusions can be drawn as to the relative contributions that the liquid and solid components each make to the robustness of a CSTR system. If the SW system were to show better performance, this would provide evidence that the maintenance of soluble compounds was most important for stable operation. The fact that the HF system showed better performance provides support for the argument that the retention of biomass was most important for stable operation.

The fraction of alkalinity that is most useful within normal operating range for anaerobic digestion is bicarbonate alkalinity, as titrated to $\mathrm{pH} 5.75$ (Ripley et al., 1986). Although bicarbonates are soluble compounds and were therefore expected to be flushed out with the liquid fraction, it was found that alkalinity could be regenerated by retaining the solid fraction, which contained active biomass and substrate. The shorter HRT also helped to prevent the build-up of VFA and ammonia, both of which are potentially inhibitory at high concentrations (Mata-Alvarez, 2003).

Although reactor designs that keep solids in the system are common for treatment of liquid waste streams, the situation is different in many commercial solid waste digestion systems, where wasting 
of solids and retention of liquids is common. Many commercial digestion facilities have a solidliquid separation step following digestion, after which the wasted solids are composted or landfilled and the liquid is recirculated to the pre-treatment system or digester (Greenfinch Ltd., 2003, Blischke, 2004). This work, however, has shown that retaining only the liquid fraction while removing solids results in accumulation of VFA and loss of biomass.

In the case of plants treating wastes with a significant fraction of materials high in lignin such as green wastes or paper, the removal of solids would be a sound strategy to avoid the build-up of materials that are resistant to degradation. In the case of highly-degradable materials, however, such as the source-separated food waste substrate used in this study, it is apparent that keeping solids in the system is more beneficial for the regeneration of alkalinity and flushing of excess VFA, and does not result in a build-up of recalcitrant materials.

These results support the use of reactor types that retain solids in the system, such as the anaerobic sequencing batch reactor (ASBR) used in the treatment of fruit and vegetable wastes by Bouallagui et al. (2005). In that study, a two-stage system was used. The two-stage mode has the advantage of regulating the loading of volatile fatty acids to the methanogenic stage, and is a strategy employed to minimise the likelihood of process upset due to rapid overproduction of VFA. Two-stage digestion has been used in many recent studies on the processing of market wastes and other food wastes containing primarily fruit and vegetables (Mtz.-Viturtia et al., 1995, Kim et al., 2000, Bouallagui et al., 2004, Parawira et al., 2004, Wang et al., 2005). In the current work, however, it was found that in the case of the HF reactors a one-stage system was sufficient to maintain stable VFA levels and a fair degree of robustness in the face of disturbance, when solids were maintained in the system while liquids were flushed through. This is a favourable result as one-stage systems are generally favoured over two-stage systems at a commercial scale, due to their lower complexity and costs; currently over $87 \%$ of $\mathrm{AD}$ plants treating the organic fraction of municipal solid waste (OFMSW) in Europe are single-stage plants (De Baere, 2006).

Single-stage digestion has been used at laboratory scale recently for feedstocks including OFMSW (Gallert et al., 2003, Bolzonella et al., 2003), wastewater and solid food wastes from food processing (Beccari et al., 1999, Carucci et al., 2005), and slaughterhouse wastes (Salminen and Rintala, 2002, Siegrist et al., 2005). This last type of waste is high in proteins and lipids, and is relevant as the food waste feedstock used in this study also has a high lipid content. The primary breakdown products of lipids are long-chain fatty acids (LCFA); in single-stage digestion, the presence of methanogens within the same stage makes the degradation of LCFA by beta-oxidation more energetically favourable by the uptake of acetate and hydrogen produced in the process (Fox and Pohland, 1994). Pereira et al. (2005) found an association of LCFA with microbial cells, and postulated that beta-oxidation may occur while LCFA is adsorbed to cells. They suggested solute transport limitations due to adsorbed LCFA as a possible mechanism for inhibition effects observed by other investigators on hydrolysis (Neves et al., 2008) and methanogenesis (Koster and Cramer, 1987, Hwu and Lettinga, 1997). Keeping cells and unhydrolysed substrate in the system with the HF mode studied would allow sufficient time for degradation of the more slowly-degradable lipids and LCFA, as well as other compounds which may be affected by mass transfer limitations resulting from LCFA adsorption.

\section{CONCLUSIONS}

In this work it was shown that keeping only the solid fraction of digestate in the reactor enabled robust and stable operation over an extended period, as well as resilience to disturbance. The flushing of liquid allowed the removal of VFA and other dissolved compounds, but sufficient 
bicarbonate alkalinity was maintained in the medium due to biomass activity. In contrast, removal of the solid fraction and extended retention of the liquid fraction resulted in accumulation of VFA and loss of methanogenic activity. Future work could be focused on decreasing the liquid retention time further, as process parameters such as VFA levels and alkalinity indicated that the system was healthy and likely could operate at a higher hydraulic flush rate.

A solids retention mode of digestion has the benefit of allowing for syntrophic relationships, while reducing the likelihood of accumulation of potentially inhibitory substances such as VFA and ammonia. This mode of digestion, therefore, is promising for application to the processing of source-separated food wastes containing a mix of readily-degradable and slowly-degradable components.

\section{ACKNOWLEDGEMENTS}

This work was supported by funding from the UK Engineering and Physical Sciences Research Council (EPSRC) Sustainable Urban Environment (SUE) Programme grant GR/S79626/01, and the UK Overseas Research Students Awards Scheme (ORSAS). The invaluable assistance of Denise Cysneiros, Mark Walker, Sonia Heaven and Yue Zhang are gratefully acknowledged.

\section{REFERENCES}

Standard Methods for the Examination of Water and Wastewater (2005). 21st edn, APHA, (American Public Health Association), American Wastewater Association and Water Environment Federation, Washington, D.C.

Beccari, M., Carucci, G., Majone, M. and Torrisi, L. (1999). Role of lipids and phenolic compounds in the anaerobic treatment of olive oil mill effluents. Environmental Technology, 20(1), 105-110.

Blischke, J. (2004). Combining Anaerobic Digestion with Enclosed Tunnel Composting. Biocycle, Apr. 2004, JG Press, Emmaus PA, USA.

Bolzonella, D., Innocenti, L., Pavan, P., Traverso, P. and Cecchi, F. (2003). Semi-dry thermophilic anaerobic digestion of the organic fraction of municipal solid waste: Focusing on the start-up phase. Bioresour. Technol., 86(2), 123-129.

Bouallagui, H., Haouari, O., Touhami, Y., Ben Cheikh, R., Marouani, L. and Hamdi, A. (2004). Effect of temperature on the performance of an anaerobic tubular reactor treating fruit and vegetable waste. Process Biochemistry, 39(12), 2143-2148.

Bouallagui, H., Touhami, Y., Cheikh, R. B. and Hamdi, M. (2005). Bioreactor performance in anaerobic digestion of fruit and vegetable wastes. Process Biochemistry, 40(3-4), 989-995.

Carucci, G., Carrasco, F., Trifoni, K., Majone, M. and Beccari, M. (2005). Anaerobic digestion of food industry wastes: Effect of codigestion on methane yield. J. Environ. Eng.-ASCE, 131(7), 10371045.

Climenhaga, M. A. and Banks, C. J. (2007). Anaerobic digestion of catering wastes: effect of micronutrients and retention time. Proceedings of the 11th IWA World Congress on Anaerobic Digestion (AD11): Bioenergy for our Future, Brisbane, Australia.

De Baere, L. (2006). Will anaerobic digestion of solid waste survive in the future? Water Science and Technology, 53(8), 187-194.

Fox, P. and Pohland, F. G. (1994). Anaerobic treatment applications and fundamentals: substrate specificity during phase separation. Water Environment Research, 66(5), 716-724.

Gallert, C., Henning, A. and Winter, J. (2003). Scale-up of anaerobic digestion of the biowaste fraction from domestic wastes. Water Research, 37(6), 1433-1441.

Gonzalez-Gil, G., Seghezzo, L., Lettinga, G. and Kleerebezem, R. (2001). Kinetics and MassTransfer Phenomena in Anaerobic Granular Sludge. Biotechnol. Bioeng., 73(2), 125-134. 
Hwu, C.-S. and Lettinga, G. (1997). Acute toxicity of oleate to acetate-utilizing methanogens in mesophilic and thermophilic anaerobic sludges. Enzyme and Microbial Technology, 21(4), 297-301. Kim, I. S., Kim, D. H. and Hyun, S. H. (2000). Effect of particle size and sodium ion concentration on anaerobic thermophilic food waste digestion. Water Science and Technology, 41(3), 67-73.

Koster, I. W. and Cramer, A. (1987). Inhibition of Methanogenesis from Acetate in Granular Sludge by Long-Chain Fatty Acids. Appl. Environ. Microbiol., 53(2), 403-409.

Lahav, O., Morgan, B. E. and Loewenthal, R. E. (2002). Rapid, Simple, and Accurate Method for Measurement of VFA and Carbonate Alkalinity in Anaerobic Reactors. Environmental Science \& Technology, 36(12), 2736-2741.

Biomethanization of the Organic Fraction of Municipal Solid Wastes (2003). 1st edn, MataAlvarez, J., TJ International Ltd., Cornwall, U.K.

Mtz.-Viturtia, A., Mata-Alvarez, J. and Cecchi, F. (1995). Two-phase continuous anaerobic digestion of fruit and vegetable wastes. Resources, Conservation and Recycling, 13(3-4), 257-267. Neves, L., Goncalo, E., Oliveira, R. and Alves, M. M. (2008). Influence of composition on the biomethanation potential of restaurant waste at mesophilic temperatures. Waste Manage., 28(6), 965-972.

Parawira, W., Murto, M., Read, J. S. and Mattiasson, B. (2004). Volatile fatty acid production during anaerobic mesophilic digestion of solid potato waste. J. Chem. Technol. Biotechnol., 79(7), 673-677.

Pereira, M. A., Pires, O. C., Mota, M. and Alves, M. M. (2005). Anaerobic biodegradation of oleic and palmitic acids: Evidence of mass transfer limitations caused by long chain fatty acid accumulation onto the anaerobic sludge. Biotechnol. Bioeng., 92(1), 15-23.

Ripley, L. E., Boyle, W. C. and Converse, J. C. (1986). Improved alkalimetric monitoring for anaerobic digestion of high-strength wastes. Journal WPCF, 58(5), 406-411.

Salminen, E. A. and Rintala, J. A. (2002). Semi-continuous anaerobic digestion of solid poultry slaughterhouse waste: effect of hydraulic retention time and loading. Water Research, 36(13), 31753182.

Sanders, W. T. M. (2001). "Anaerobic hydrolysis during digestion of complex substrates," Wageningen Agricultural University, Wageningen.

Sanders, W.T.M., Veeken, A., Zeeman, G., van Lier, J.B. (2003). Analysis and optimisation of the organic fraction of municipal solid waste. In: Biomethanization of the Organic Fraction of Municipal Solid Wastes (2003). 1st edn, Mata-Alvarez, J., TJ International Ltd., Cornwall, U.K. Siegrist, H., Hunziker, W. and Hofer, H. (2005). Anaerobic digestion of slaughterhouse waste with UF-membrane separation and recycling of permeate after free ammonia stripping. Water Science and Technology, 52(1-2), 531-536.

Veeken, A., Kalyuzhnyi, S., Scharff, H. and Hamelers, B. (2000). Effect of pH and VFA on Hydrolysis of Organic Solid Waste. Journal of Environmental Engineering, 126(12), 1076-1081. Wang, J. Y., Zhang, H., Stabnikova, O., Ang, S. S. and Tay, J. H. (2005). A hybrid anaerobic solidliquid system for food waste digestion. Water Science and Technology, 52(1-2), 223-228. 